\title{
Tangential curved planar reformation for topological and orientation invariant visualization of vascular trees
}

\author{
Noah Lee and Matthias Rasch
}

\begin{abstract}
Recently, extensions to curved planar reformation (CPR) were proposed to improve vascular visualization of medical images. While these projective transformations provide enhanced visualization of vascular trees, non-planar alignment and arbitrary topology can cause visualization artifacts. Vascular trees in medical images are not aligned to planar cross-sections of volumetric image slices and thus aggravate simultaneous visualization of diagnostic features. Complex tree topology and non-planar alignment requires the need for an adaptive projection scheme to prevent visualization artifacts while preserving correctness of anatomical information. In this paper, we present algorithmic details for topological and orientation invariant visualization of vascular trees. Vascular high-level description of the medial axis guides the reformation process by flattening the vascular tree interior to successive image planes for respective radial sampling angles. Tree orientations are estimated from intrinsic shape properties of the vascular tree for rotation invariant projection. Radial sampling planes perpendicular to the medial axis tangents are the basis for topological invariant visualization of complete vascular interiors. We present experimental results on two different vascular tree topologies and demonstrate that our method is able to produce artifact free visualization of vascular interiors.
\end{abstract}

\section{INTRODUCTION}

Vascular imaging using computed tomography angiography (CTA) or magnetic resonance angiography (MRA) are non-invasive high-resolution in-vivo image acquisition techniques for evaluating vascular diseases such as coronary artery disease (CAD), peripheral artery disease (PAD), and derivatives. In particular, these modalities provide highresolution information about vascular pathology such as stenosis, calcification, and atherosclerotic plaque. The vessel interior is of great importance for evaluating the degree and distribution of vascular diseases. In order to evaluate the vascular tree the whole vessel lumen must be investigated, which is tedious and time consuming [1]. Visualization techniques namely curved planar reformation (CPR) re-sample a longitudinal cross-section along the medial axis in one image plane for simultaneous and accurate visualization of diagnostic features. Recently, extensions to curved planar reformation (CPR) were proposed to improve visualization of complete vascular trees. While these projective transformations provide enhanced visualization of vascular trees, nonplanar alignment and arbitrary topology can cause visualization artifacts.

Noah Lee is a graduate student of Biomedical Engineering, Columbia University, Heffner Biomedical Imaging Laboratory, 1210 Amsterdam Ave., New York, NY 10027, USA.nl2168@columbia.edu

Matthias Rasch is with Siemens Corporate Research, Imaging an Visualization Dept., College Road East 755, Princeton, NJ 08540-6632, USA.rasch@scr.siemens.com
Vascular trees in medical images are not aligned to planar cross-sections of volumetric image slices and thus aggravate simultaneous visualization of diagnostic features. Furthermore, complex tree topology requires the need for an adaptive projection scheme to prevent visualization artifacts while preserving correctness of anatomical information.

In this paper, we present algorithmic details for topological and orientation invariant visualization of complete vascular trees. Vascular high-level description of the medial axis are obtained within an energy optimization framework utilizing front propagation and graph-theoretic approaches. The medial axis guides the reformation process by mapping the vascular tree to successive image planes for respective radial sampling angles. Tree orientations are estimated from intrinsic shape properties of the vascular tree for rotation invariant projection. Radial sampling planes perpendicular to the medial axis tangents are the basis for topological invariant visualization of complete vascular interiors. We present experimental results on two different vascular tree topologies and demonstrate that our method is able to produce artifact free visualization.

\section{RELATED WORK}

Little has been published on technical aspects of automated techniques for visualizing the interior of vascular trees with arbitrary topology and orientation. Regarding vascular visualization a pool of techniques exist such as multi-planar reformation (MPR), shaded surface display (SSD), maximum intensity projection (MIP), curved MPRs, or volume rendering (VR). However, diagnostic features of vascular trees with small scale diameter such as in coronary arteries are often hard to investigate simultaneously with aforementioned visualization techniques. In VR, atherosclerotic plaque accumulating on vessel walls can obstruct the view of vascular interior crucial for disease diagnosis. Furthermore, other body tissues often occlude the objects of interest while traditional MPR views only visualizes fractions of the complete vascular tree due to non-planar alignment. Manual creation of curved MPRs for whole vascular trees is tedious and time consuming especially in large patient studies. Other methods for automatic generation of curved planar reformations only consider a single vessel segment to visualize [1, 2]. Vrtovec et al. applied CPR on 3D spine images for automated visualization [3]. Kanitsar et al. proposed extensions to traditional CPR techniques consisting of "multi-path" and "rotated" CPR techniques [4]. They also proposed two advanced CPR techniques called "helical" and "untangled" CPR enabling the visualization 
of complete vascular interiors for single vessel segments in one image and occlusion free reformation of the whole vascular tree using an untangling scheme [5]. However, to deal with arbitrary topology and non-planar alignment these projective transformations cannot directly be applied. Complex tree topology with high vessel curvature require an adaptive projection scheme with tangential aligned radial sampling planes for artifact free visualization. In our work we describe solutions and implementation details to the problem of handling arbitrary topology and orientations of vascular trees and apply our projection scheme to coronary artery and peripheral artery trees. We will denote our projection scheme as tangential curved planar reformation (TCPR).

\section{Methodology}

\section{A. Implicit Tree Representation of Medial Manifold}

Let's assume $M_{T}$ represents a discrete connected subset in domain $\Omega \subset \Re^{3}$ that represents an anatomical manifold of a binary tree with arbitrary topology and orientation. $M_{T}$ is binarized such that $M_{T}(\phi)=1$ inside and $M_{T}(\phi)=0$ outside the manifold. Furthermore, lets assume this manifold is topological correct, i.e. does not contain holes, is closed, and differentiable. We seek the medial path $M_{P} \subset M_{T}$, by maximizing the energy functional in equation (1), such that $M_{P}$ fulfills the following properties of medialness: 1) $M_{P}$ is connected, 2) one-voxel thick, and 3) reconstructable i.e. the transformation $\Psi: M_{T} \mapsto M_{P}$ is bijective.

$$
E(\phi)=\int_{\Omega} f\left(M_{T}(\phi)\right)+\left(1-\left|\frac{\partial M_{T}}{\partial \phi}\right|\right) d \Omega
$$

With medial we mean, that the manifold subset $M_{P}$ is centered with respect to the boundary of $M_{T}$ and is uniquely defined. To maximize equation (1) we perform a two-stage process. First, we label $M_{T}$ with an Euclidean distance function $f$ from the boundary set $M_{B}=\left\{\phi \in M_{B} \mid N_{6}(\phi) \leq N_{\max } \wedge\right.$ $\left.N_{6}(\phi)>0\right\}$ with $N_{6}(\phi)=\left\{\phi^{\prime} \in Z^{3}\left|\sum\right| \phi_{i}-\phi_{i}^{\prime} \mid \leq 1\right\}$ and $N_{\max }=6$. From $M_{B}$ we propagate a front with constant speed $s$ in the direction of the boundary inward normal, whos arrival time $t$ labels $M_{T}$, such that the Eikonal equation is satisfied:

$$
\left|\nabla M_{T}\right|=\sum_{i=1}^{n}\left(\frac{\partial M_{T}}{\partial \phi_{i}}\right)^{2}=1
$$

Since $s=$ const., arrival time $t$ is a measure for medialness of $M_{P}$. Second, to satisfy property of medialness 1) and 2) let $G=(V, E)$ be a connected, undirected, weighted graph of $M_{T}$ with a function $w: E \mapsto \Re$, that assigns a weight $w(e)$ to each edge $e$. We want to find a subset of $E^{\prime} \subset$ $E$ of minimum weight forming a tree $T$ on $V$, such that $G$ remains a one connected component. Thus, we form a minimum spanning tree $T$ of $G$, minimizing the weighting function

$$
w(T)=\sum_{e \in T} w(e)=-f\left(v_{i}\right)+\left|v_{i}-v_{i-1}\right|, v_{i} \in V .
$$

For $w(e)$ we use two distance metrics: i) negative medialness $f$ computed after equation (2), and ii) the geodesic distance in the topological neighborhood $N_{26}$, with $N_{26}(\phi)=\left\{\phi^{\prime} \in\right.$ $\left.Z^{3} \mid \max \left(\left|\phi_{i}-\phi_{i}^{\prime}\right|\right) \leq 1\right\}$. Note that the edge weights must be distinct i.e. $w(e) \neq w\left(e^{\prime}\right)$ for any pair of edges $e$ and $e^{\prime}$, so $T$ is unique. For obtaining $T$ from $G$ we use Kruskal's algorithm. To reduce tree complexity of $T$ we perform recursive node abstraction, where $T$ is simplified into a binary node tree, till all non-end nodes $N_{i}^{*} \in N_{i}$ are bi-furcation nodes and each node $N_{i} \in T$ stores its parent connecting path $p_{i-1}$ to $N_{i-1}$. The order of $T$ is recursively reduced until valence $\left(N_{i}^{*}\right)=3, \forall N_{i}^{*}$. In what follows, we will denote this abstract solution as $T^{\prime}$.

\section{B. Intrinsic Orientation Determination and Adjustment}

As noted before, $T^{\prime}$ can be oriented arbitrarily due to nonplanar alignment, thus no assumption can be made regarding the axis of projection vector $\vec{v}$ that will be used for tree projection in Section $C$. To be invariant against arbitrary tree orientations, we change the viewing basis to an orthonormal basis aligned to the intrinsic shape of the vascular tree. To determine $\vec{v}$ we compute the center of mass $\mu_{\phi}$ of $T^{\prime}$ defined as

$$
\mu_{\phi}\left(T^{\prime}\right)=\int_{\Omega} x n \phi d x, \text { with } n \phi=\frac{N \phi}{\int_{\Omega} N \phi d x} .
$$

Then, $\vec{v}$ is defined as the direction vector pointing from $\mu_{\phi}$ to $N_{0}$, i.e. $\vec{v}=n_{0_{i}}-\mu_{\phi_{i}}=$ $\left\langle n_{0_{1}}-\mu_{\phi_{1}}, n_{0_{2}}-\mu_{\phi_{2}}, n_{0_{3}}-\mu_{\phi_{3}}\right\rangle$, with $N_{0}$ being the root node of $T^{\prime}$. Note that this approach is robust against ambiguous shape topology, which causes problems in PCA based methods. Remembering the property of reconstructability of $M_{T}$, the vector $\overrightarrow{n_{0}}$ is the position vector of node $N_{0}$ with maximum value of medialness $f\left(M_{P}\right)$ computed after equation (2). The assumption of $N_{0}=\max \left(f\left(N_{i}\right)\right)$ as being the root of $T^{\prime}$ is safe, since branch diameter of anatomical trees decrease with its branching depth level. With $\vec{v}$ defined, before we can project $M_{T}$ onto $\Re^{2}$ we need a continuous representation of $M_{P}$ for reformation and resampling. Therefore, every path in $N_{i}$ is re-sampled using B-Spline interpolation.

\section{Vascular Tree Projection from $\Re^{3}$ to $\Re^{2}$}

We project $T^{\prime}$ from $\Re^{3}$ to $\Re^{2}$ and align $T^{\prime}$ such that the projection of $T^{\prime}$ is oriented top-to-bottom. Furthermore, we project $M_{P}$ at different viewing angles $\theta \in[0 \ldots 2 \pi]$, where each projection is stored in corresponding image planes $C_{\theta}$. Consider the sets of lines $L=\left\{L_{i}\right\}$ emanating from the nodes $N=\left\{N_{i}\right\}$ such that their normalized direction vectors fulfill $\left\langle\overrightarrow{l_{i}} \mid \overrightarrow{v_{l}}\right\rangle=0$, where $\overrightarrow{v_{l}}=\left\langle\mu_{\phi_{1}}, \mu_{\phi_{2}}, \mu_{\phi_{3}}\right\rangle+t\langle a, b, c\rangle$. The coefficients $a, b$, and $c$ denote the normalized direction vector of $\vec{v}$. Let $\overrightarrow{v_{l}}$ be a line congruent to $\vec{v}$, then the maximum node-to-line distance $d_{n l}$ from $N_{i}$ to $\overrightarrow{v_{l}}$ is

$$
\begin{gathered}
\Delta x=x_{0}-x_{1}, \Delta y=y_{0}-y_{1}, \Delta z=z_{0}-z_{1} \\
A=\left[\begin{array}{cc}
\Delta y & \Delta z \\
b & c
\end{array}\right], B=\left[\begin{array}{cc}
\Delta z & \Delta x \\
c & a
\end{array}\right], C=\left[\begin{array}{cc}
\Delta x & \Delta y \\
a & b
\end{array}\right]
\end{gathered}
$$




$$
d_{n l=\max }\left\{\sqrt{\frac{|A|^{2}+|B|^{2}+|C|^{2}}{a^{2}+b^{2}+c^{2}}}\right\}
$$

and $\omega$ the maximum branch diameter of $M_{T}$ is $\omega=2$. $\max \left(f\left(M_{P}\right)\right)$. The plane-to-axis of projection distance $d_{p a}$ is the sum of $d_{n l}$ and $\omega$. We build a set of projection planes $P_{\theta}$ and compute the minimum node-to-projection plane distance $d_{n p} . P_{\theta}$ is spanned around $T^{\prime}$ to compute relative projection coordinates with respect to the axis of projection $\vec{v}$. For each projection at angle $\theta$, we project $M_{P}$ onto image plane $C_{\theta}$ by using two distance measures: 1) the projected x-translation in $\Re^{3}$ being $d_{n p}$ and 2) the projected $\mathrm{y}$-translation being the parameterized arc length $l_{c}$ defined as follows:

$$
\begin{array}{r}
\delta x=\frac{\left|A\left(x_{1}-x_{0}\right)+B\left(y_{1}-y_{0}\right)+C\left(z_{1}-z_{0}\right)\right|}{\sqrt{A^{2}+B^{2}+C^{2}}} \\
\delta y=\int_{0}^{t} d s, d s=\sqrt{\left(\frac{d x}{d t}\right)^{2}+\left(\frac{d y}{d t}\right)^{2}+\left(\frac{d y}{d t}\right)^{2}} d t .
\end{array}
$$

Here $\delta x$ and $\delta y$ denote coordinates of the projected medial path $M_{P}$ onto $C_{\theta}$ for each projection angle $\theta$ respectively.

\section{Topological Invariant Tangential Re-Sampling}

We compute the Frenét trihedral for the set of vector functions $R=\left\{\vec{r}_{i}(t)\right\}$ representing individual curve segments stored in all non-end nodes $N_{i} \in T^{\prime}$, with $i>0$. The set of curves satisfies the following conditions: 1$) \vec{r}_{i}(t)$ is open and 2) $\vec{r}_{i}^{\prime \prime}(t)$ exist. Then we sample along $\vec{r}_{i}(t)$ by defining a set of sample lines $L=\left\{L_{\theta}\right\}_{\theta=0}^{2 \pi}$ for each point $p$ in $\vec{r}_{i}(t)$ such that $L$ lies in a sample plane $\mathrm{P}$ perpendicular to $r_{i}^{\prime}(t)$. The sample plane is spanned by $\hat{t}(t)$ and $\hat{n}(t)$.

$$
\begin{aligned}
& \hat{t}(t)=\frac{\vec{r}^{\prime}(t)}{\left\|\vec{r}^{\prime}(t)\right\|}, \hat{n}(t)=\frac{\hat{t}^{\prime}(t)}{\left\|\hat{t}^{\prime}(t)\right\|}, \hat{b}(t)=\hat{t}(t) \times \hat{n}(t) \\
& {\left[\begin{array}{ccc} 
& R(\hat{a}, \theta)= \\
s x^{2}+\cos (\theta) & s x y-\sin (\theta) z & s x z+\sin (\theta) y \\
s x y+\sin (\theta) z & s y^{2}+\cos (\theta) & s y z-\sin (\theta) x \\
s x z-\sin (\theta) y & s y z+\sin (\theta) x & s z^{2}+\cos (\theta)
\end{array}\right]} \\
& \text { with } s=1-\cos (\theta), \hat{a}=\langle x, y, z\rangle \\
& l_{\theta}=\left\{\left\langle p_{x}, p_{y}, p_{z}\right\rangle+t\left\langle a_{\theta}, b_{\theta}, c_{\theta}\right\rangle\right\}_{\theta=0}^{2 \pi}, t \in\left[d_{n l},-d_{n l}\right] \\
& \left\langle a_{\theta}, b_{\theta}, c_{\theta}\right\rangle=R(\hat{t}(t), \theta) \cdot \hat{b}(t)
\end{aligned}
$$

We rotate $\hat{b}(t)$ around $\hat{t}(t)$ using equation (9) by building corresponding sampling lines $l_{\theta}$ for tangential data sampling. Note that $l_{\theta}$ samples a complete cross section of the lumen at $r_{i}(t)$ and since $L$ is perpendicular to $r_{i}^{\prime}(t)$ visualization of vessel interiors are invariant to arbitrary tree topologies. The final step consists of mapping our samples to consecutive image planes, where the first phase denotes a flattened tree at projection angle $\theta=0$.

\section{E. Image Space Partitioning and Artifact Free Rendering}

The projected tree $T^{\prime} \subset \Re^{2}$ computed in Section $C$ is used to define a partition of each phase $C_{\theta}$ based on following algorithmic scheme. For each $C_{\theta}$ we map the projected tree coordinates $\delta x$ and $\delta y$ computed in equation (6) and (7) and label each phase according to:

$$
\begin{gathered}
C_{\theta}(x, y)=\left\{\begin{array}{cc}
P_{i}(t) & \text { if } C_{\theta}(x, y)==0 \\
P_{j}(t) & \text { if } a<b \\
0 & \text { else }
\end{array}\right. \\
\text { with } a=\operatorname{dist}\left(\text { obs }, P_{j}(t)\right), b=\operatorname{dist}\left(\text { obs }, P_{i}(t)\right),
\end{gathered}
$$

where obs is the virtual camera observer in $\Re^{3}$ and $P_{i}$ and $P_{j}$ are two intersecting projected curves in $C_{\theta}$. The next step is to create an image partitioning based on following rule set:

$$
R_{\theta}=\bigcup_{i=1}^{n} R_{i}=\int_{P_{i}(0)}^{P_{i}(m-1)} \int_{P_{i}(y)}^{P_{j}(y)} f(\phi) d \Omega
$$

where a distance function $f$ is computed from each point $C_{\theta}>0$ outward, and each region $R_{i}$ associated to a path $P_{i}$ is computed by maximizing the energy potential function in the bounds of equation 13. Finally, we fill each region $R_{i}$ with sample data obtained in Section D by using following line rendering scheme:

$$
\begin{aligned}
& \text { Let } D=\left\{p \in R^{2} \mid\left(p_{i} \in P_{i}\right)=\left(p_{k} \in P_{k}\right)\right\} \\
& \forall P_{i} \in T^{\prime} \text { in } \Re^{2} \text { and } i=N-1\{ \\
& x_{l}=\{0\}_{j=0}^{j=s i z e}\left(P_{i}\right)-1, x_{r}=x_{l} \\
& \forall p_{j} \in P_{i}\left\{\begin{array}{c}
\left\{=\text { with } k=\left\{c, c+\operatorname{size}\left(L_{i}\right)\right\}\{\right. \\
\forall p_{j, k} \in L_{i} \text { with } \\
\left.\left.\exists p_{j, k} \in D\right)=p_{j} \wedge j>0\right) \\
i f\left(x_{r}\{i-1\}>0 \wedge x_{r}\{i\}==0\right) \\
x_{r}\{i\}=x_{r}\{i\}+1 ; \text { continue; } \\
i f\left(\left(\exists p_{j, k} \in C_{\theta}(x, y)>0\right)\right) \\
x_{r}\{i\}=x_{r}\{i\} / 2 ; \\
x_{r}\{i\}=\operatorname{ceil}\left(x_{r}\{i\}\right)-1 ; \text { break; } \\
x_{r}\{i\}=x_{r}\{i\}+1 ;
\end{array}\right.
\end{aligned}
$$

This scheme prevents image artifacts in form of toggle line renderings due to discretization in the reformation process. We note that above algorithm describes the line rendering scheme only for the right side of a region, but can be easily adopted for the left side due to symmetry.

\section{EXPERIMENTAL RESUlts}

Experiments on two different vascular tree anatomies have been performed: 1) coronary and 2) peripheral vascular trees. Coronary trees are highly curved and comprise arbitrary topology and non-planar orientation. Segmentation masks of vascular trees were obtained from CTA and MRA datasets using a model-based vessel segmentation algorithm. Segmentation masks were binarized and inputted into our visualization algorithm. Visualization results on a coronary artery tree are shown in part B and D and were obtained automatically. Comparison between non-tangential CPR and our method are shown in part A and B. Visualization results on a two peripheral trees are shown in part $\mathrm{C}$. The goal was 

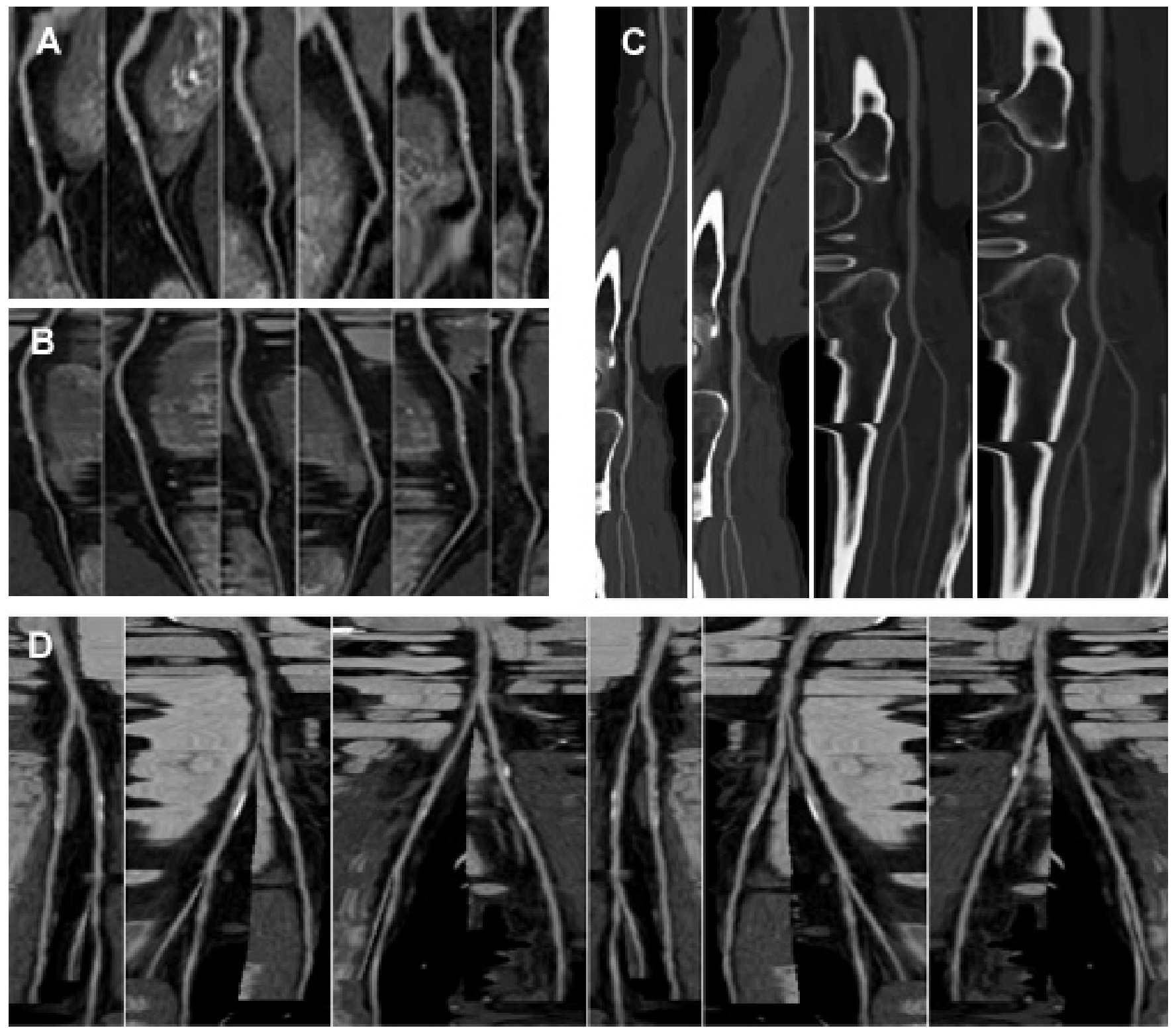

Fig. 1. Experiments using TCPR. (A, B) Comparison between non-tangential and TCPR projection scheme (MRA). Note sampling artifacts on A whereas using our TCPR scheme visualizes correct anatomic information. (D) Vascular tree visualization using TCPR on two different peripheral artery trees (CT). (D) Vascular tree visualization using TCPR on coronary artery tree at different radial sampling angles (CTA).

to visualize the whole vessel tree in one image plane while being able to show the whole vessel lumen throughout the vascular tree. We found that tangential curved planar reformation (TCPR) is able to produce artifact free visualization of vascular tree interiors. The reformation procedure does not require any user intervention and is computationally efficient.

\section{CONCLUSION}

We have presented a method for topological and orientation invariant vascular tree visualization by exploiting intrinsic shape properties of the vascular tree for rotation invariant projection and radial sampling planes perpendicular to the medial axis tangent. One limitation of TCPR is the loss of surrounding tissue information context, however experimental results confirm that complete vascular interiors can be visualized accurately without artifacts due to complex tree topology or non-planar orientation. We have presented several visualization experiments to corroborate the theory.

\section{REFERENCES}

[1] R. Raman, S. Napel, C.F. Beaulieu et al. "Automated Generation of Curved Planar Reformations from Volume Data: Method and Evaluation". Radiology 2002. 223:275-80.

[2] L. Saroul et al. "Exploring Curved Anatomic Structures with Surface Sections". IEEE Visualization 2003, Seattle, Washington, USA

[3] T. Vrtovec, B. Likar, and F. Pernus. "Automated curved planar reformation of 3D spine images". Phys. Med. Biol. 50: 4527-4540. 2005.

[4] A. Kanitsar et al. CPR - Curved Planar Reformation. IEEE Visualization 2002, Bosten USA.

[5] A. Kanitsar et al. Advanced Curved Planar Reformation: Flattening of Vascular Structures. IEEE Visualization 2003. Seattle, Washington, USA. 